\title{
Determination of best regime for administration of atropine eye drops for cycloplegia
}

\begin{abstract}
Purpose: To determine the best regime for administration of atropine eye drops for cycloplegia and to see the effect of cycloplegia on different types of refractive error.

Methods: This cross-sectional study was conducted in Pediatric Clinic, Mayo Hospital Lahore, Pakistan in the duration of 3 months to assess the best regime for administration of $1 \%$ atropine eye drops for cycloplegia and to see the effect of cycloplegia on different types of refractive error. Non probability purposive sampling was done and 30 patients of age between 0 to 12 years were assessed using a self designed performa. Patients who required cycloplegic refraction were examined after installation of atropine 1 drop for 1 day and 3 drops for 3 days by auto refactometer and retinoscopy. Results were compared to find out the difference in refractive error and the effect of cycloplegia on myopes and hyperopes.
\end{abstract}

Results: The results showed that most of the patients had no difference in refractive error between one day of atropine instillation as compared to three days. Few patients showed little difference of $+/-0.25 \mathrm{D}$ after three days. Hyperopic patients showed less difference in refractive error found between first and third day of atropine instillation as compared to myopic patients.

Conclusion: Atropine is an efficient cycloplegic drug and we can get effective cycloplegia on first day with even one drop of atropine instillation. This may be beneficial for both the practitioner and children while doing cycloplegic refraction. It will greatly reduce the waiting time for parents and children. It will also lessen the adverse effects of atropine and allow for a better clinical service.

Keywords: best regime, atropine eye drops, cycloplegia, refractive error, accommodative power
Volume 6 Issue I - 2017

\author{
Adnan Afsar,' Javaria Asif Bajwa ${ }^{2}$ \\ 'Optometry scholar, Pakistan Institute of Community \\ Ophthalmology, Pakistan \\ ${ }^{2}$ Optometry scholar, King Edward Medical University, Pakistan
}

Correspondence: Adnan Afsar, Optometry scholar, Pakistan Institute of Community Ophthalmology, A I O, Sardar Ahmad Jan colony Peshawar, Pakistan, Tel +0923349092917,

Email adnanafsar55@gmail.com

Received: August 26, 2016 | Published: January 26, 2017

\section{Introduction}

The eye is like a camera, having an optical system starting from the tear film, cornea, aqueous humor, lens, vitreous humor and retina. When the optical power and the axial length of the eye conjugate, the image of an object is focused on the retina. Refractive error is a very common disorder of eye; it occurs when the eye cannot focus light rays entering from infinity on the retina with accommodation being at rest. These errors may be classified into different categories i.e. myopia, hyperopia and astigmatism. In Myopia one feels difficulty while seeing distant objects. In Hyperopia one cannot see near objects clearly. In Astigmatism, there are two focal points which cause distorted vision. Uncorrected refractive errors are frequent cause of reduced vision in children so it is very important for children to have an eye examination. Symptoms include blur vision, squeezing the eyes, eye strain, headache etc. Some children can present with strabismus. ${ }^{1}$

The prevalence rate of visual impairment caused due to uncorrected refractive errors is estimated to be 153 million people, of whom eighty lacs are blind. ${ }^{2}$ Refractive errors can be diagnosed by vision examination and may be corrected with glasses and contact lenses. ${ }^{3}$ High level of accommodative power in children affects the refractive status of the eye as lens' optical power and shape can be altered by contracting ciliary muscles. Therefore, it is necessary to relax the accommodation fully while assessing their refractive error.
In this regard cycloplegic drugs are instilled in eyes for accuracy in measurements. Cycloplegic drugs produce paralysis of ciliary muscles which is called cycloplegia. These drugs can be instilled in the conjunctival sac, such as atropine, homatropine, tropicamide, scopolamine and cyclopentolate, which are known as cycloplegics. By paralyzing the parasympathetic nerve supply all accommodation can be abolished and latent refractive errors become manifest.

Cyclopentolate produces a rapid and intense mydriasis and satisfactory cycloplegia. Paralysis of accommodation and full dilatation of pupil are usually completed within 1 hour and the effects worn off within 24 hours; occasionally the duration of action is much longer, even 3 or 4 days. ${ }^{4}$ Atropine is an anti-cholinergic drug used to temporarily paralyze accommodation reflex in children and also as a mydriatic to dilate pupil. It is the most efficient cycloplegic agent. Although it is considered a relatively safe drug, some local and systemic side effects do occur. The recommended regimen for atropine Cycloplegia has been 7 to 10 applications within 3 to 4 days. ${ }^{5}$ The purpose of this study is to find out the best regime for atropine instillation to achieve maximum cycloplegia. It will benefit both the practitioner and children while doing Cycloplegic refraction.

\section{Methods}

This is a cross sectional study. Patients who presented at paediatric ophthalmology department Mayo Hospital Lahore, Pakistan were 
selected to assess the best regime for administration of $1 \%$ atropine eye drops for cycloplegia and to see the effect of cycloplegia on different types of refractive error. Non probability purposive selection was used to identify the study sample. After finalizing the sample size all data was collected through self designed performa. 30 patients of age between 0 to 12 years who required cycloplegic refraction were examined after instillation of atropine eye drops, one drop for one day and three drops for three days by auto refactometer and retinoscopy. Results were compared to find out the difference in refractive error and the effect of cycloplegia on myopes and hyperopes. To achieve the objectives of the study the data was collected in a period of three months. All the data was entered and analyzed using Statistical Package for Social Science (SPSS Version 20.0). All the data was presented in forms of graphs (Graph1-4).

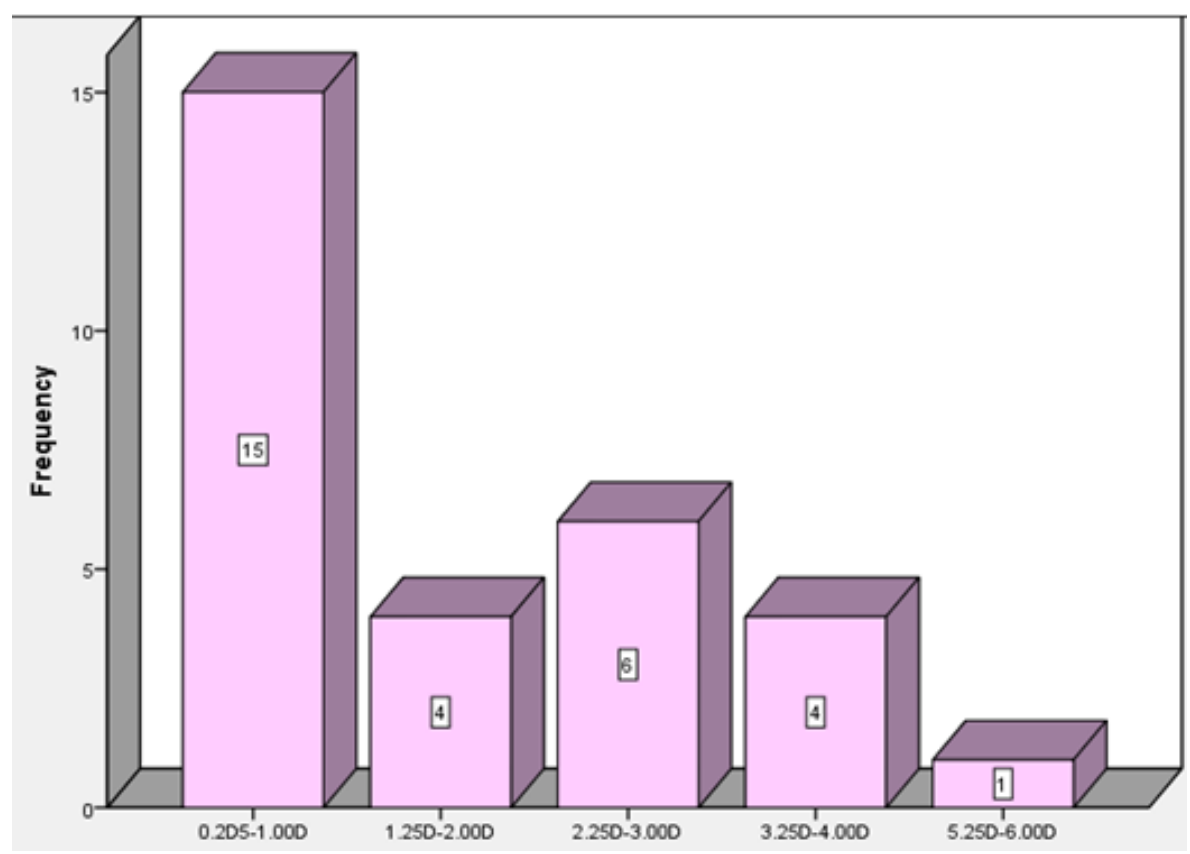

Graph I Day I spherical equivalent of refraction with I drop of atropine.

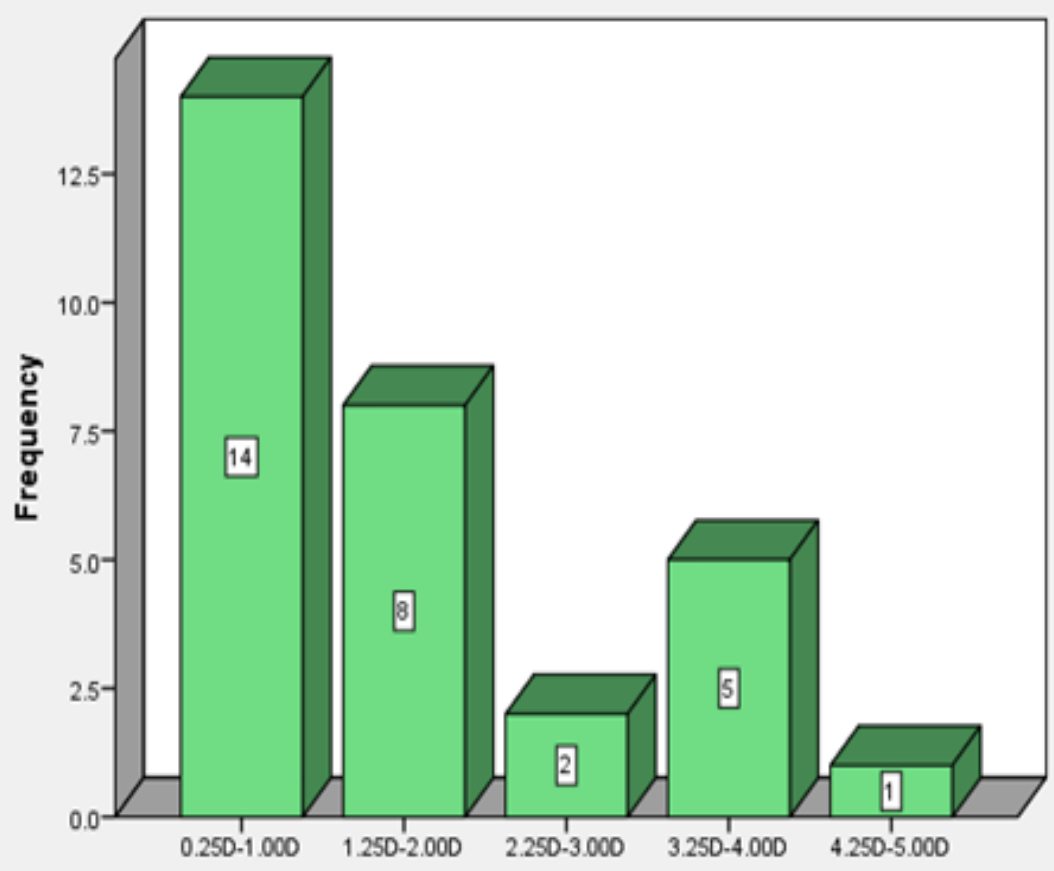

Graph 2 Day 3 spherical equivalent of refraction with 3 drops of atropine. 


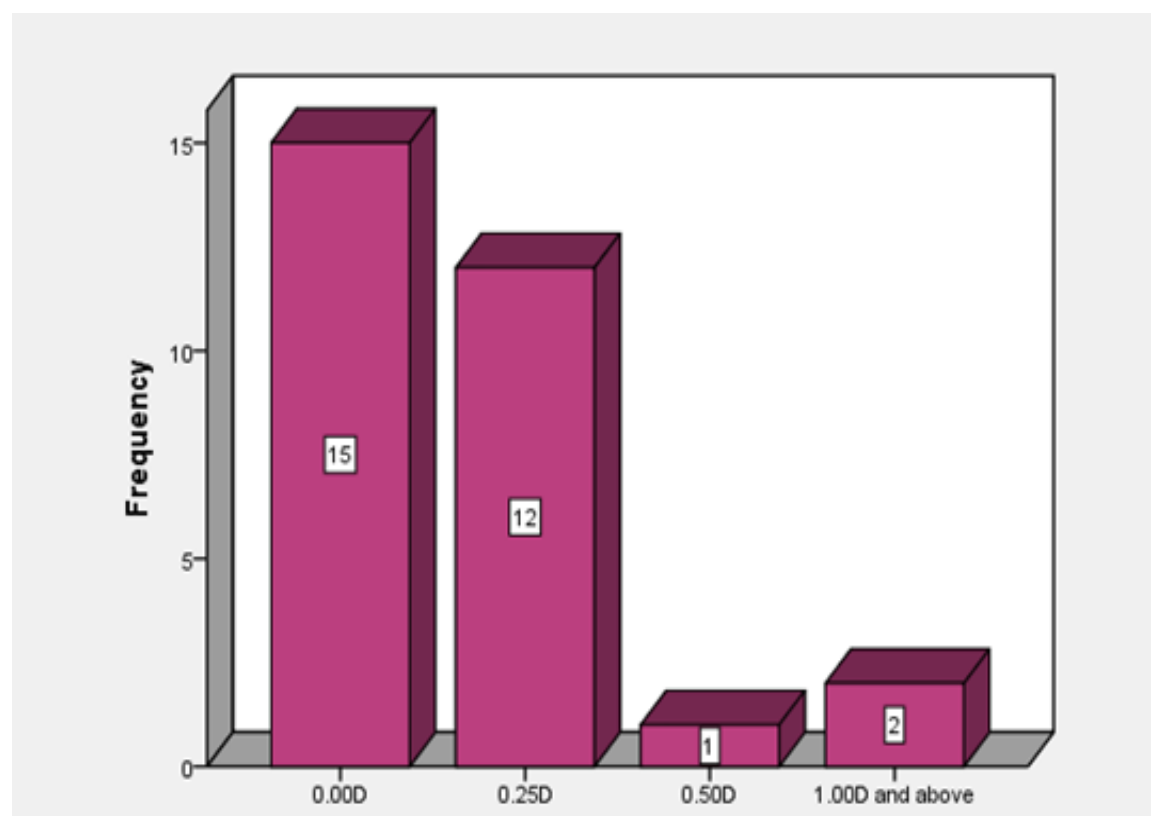

Graph 3 Difference of refractive error found at dayl and day 3 refraction.

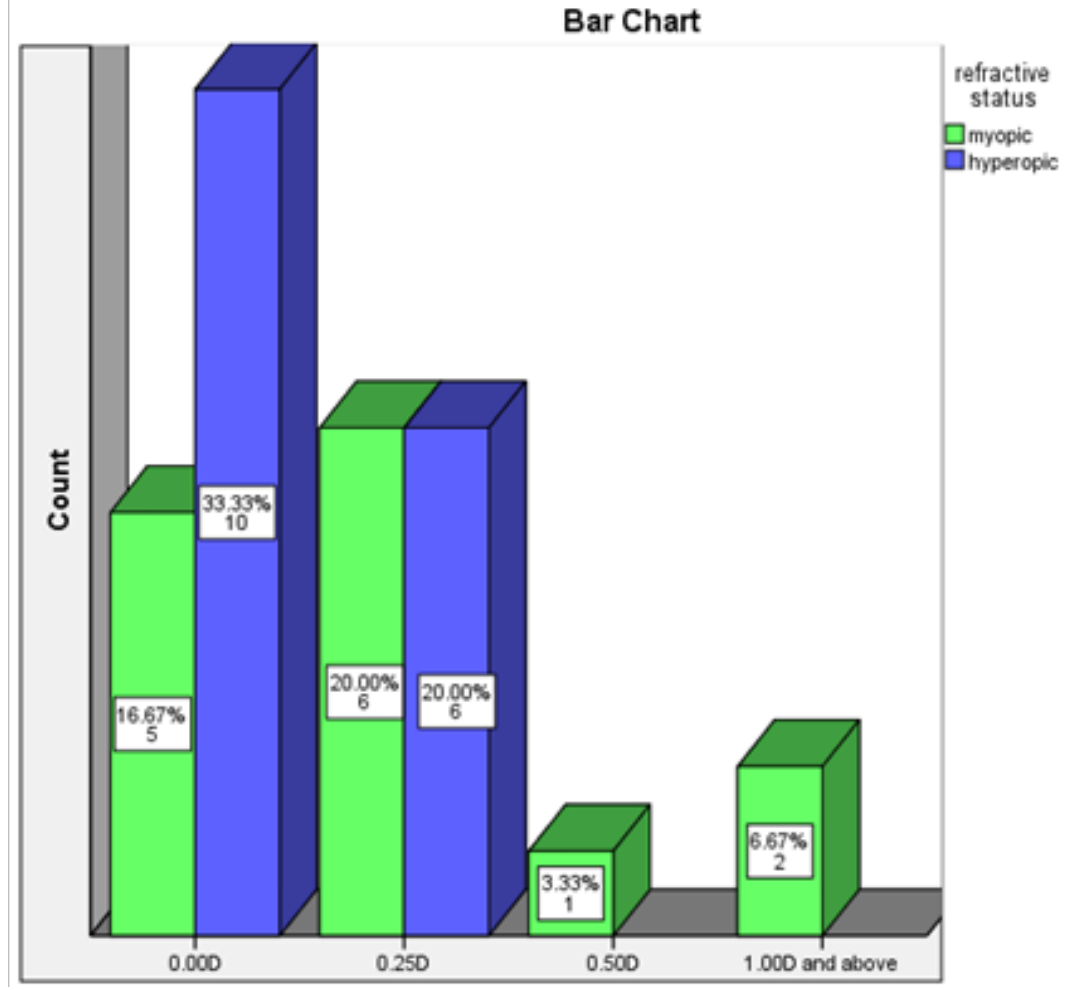

Graph 4 Difference according to type of refractive error.

\section{Results}

Mostly patients were in the age groups of 4 to 9 years. There were 18 females and 12 males. $55.33 \%$ patients were hyperopic and $46.67 \%$ were myopic. The results I found showed most of the patients have no difference in refractive error between one day of atropine instillation as compared to three days. Few patients showed little difference of $+/-0.25 \mathrm{D}$ after three days. Hyperopic patients showed less difference in refractive error found between first and third day of atropine instillation as compared to myopic patients. Mostly patients had spherical equivalent within the range of 0.25 to $1.00 \mathrm{D}$ with one drop of atropine instillation and in some patients' refractive errors found in the range of 1.25 to 4.00 Diopters.

Mostly patients had spherical equivalent within the range of 0.25 Diopters to $1.00 \mathrm{D}$ with three drops of atropine instillation after three days. Some patients also showed increase in refractive error at third day in the range of 1.25 to $2.00 \mathrm{D}$ and 3.25 to 4.00 Diopters. Most of 
the patients showed no difference in refractive error between first day of atropine instillation and after 3 days. Some patients showed little difference of $0.25 \mathrm{D}$ after three days and very few showed up to 1 D difference. Hyperopic patients showed less difference in refractive error found between first and third day of atropine instillation.

\section{Discussion}

As high level of accommodation in children affects the refractive status of eye so it is necessary to relax it fully while assessing their refractive error. In this regard Cycloplegic drugs are instilled in eyes for accuracy in measurements. ${ }^{6}$ Common Cycloplegic drugs are atropine, cyclopentolate, tropicamide, homatropine and scopolamine. ${ }^{7}$ Atropine is an anticholinergic drug used to temporarily paralyze accommodation reflex in children and also as a mydriatic to dilate pupil. ${ }^{8}$ This study was conducted to determine the best regime for administration of $1 \%$ atropine eye drops for cycloplegia by comparing the efficacy of 1 day administration of atropine eye drops with 3 days total instillation dose and to compare the effect of atropine on myope and hypermetropes.

In this study 30 patients were assessed. The results showed most of the patients have no difference in refractive errors between first day of atropine instillation and after 3 days. Few patients showed little difference of $+/-0.25 \mathrm{D}$ after three days. Hyperopic patients showed less difference in refractive error found between first and third day of atropine instillation as compared to myopic patients. Few studies were conducted to find the comparison between efficacy of different cycloplegic agents in children ${ }^{9}$ and use of atropine for the treatment of myopia ${ }^{10}$ but there is lack of literature on best regime of atropine administration for cycloplegia. This study was specifically conducted on dark iris children by comparing the difference of first and third day refractive error and between 1 and 3 drops instillation.

\section{Conclusion}

Atropine is an efficient cycloplegic drug and we can get effective cycloplegia on first day with even one drop of atropine instillation. This study may be beneficial for both the practitioner and children while doing cycloplegic refraction. It will greatly reduce the waiting time for parents and children. It will also lessen the adverse effects of atropine and allow for a better clinical service.

\section{Funding}

None.

\section{Acknowledgments}

None.

\section{Conflicts of interest}

The authors declare that there was no conflict of interest.

\section{References}

1. Zhu H, Yu JJ, Yu RB, et al. Association between childhood strabismus and refractive error in Chinese preschool children. PloS one. 2015;10(3):e0120720.

2. Resnikoff S, Pascolini D, Mariotti SP, et al. Global magnitude of visua impairment caused by uncorrected refractive errors in 2004. Bull World Health Organ. 2008;86(1):63-70.

3. Dandona R, Dandona L, Srinivas M, et al. Refractive error in children in a rural population in India. Invest Ophthalmol Vis Sci. 2002;43(3):615-622.

4. Ward PA, Charman WN. Measurements of cycloplegia and mydriasis induced by three common ophthalmic drugs. Clinical and Experimental Optometry. 1986;69(2):62-70.

5. Stolovitch C, Loewenstein A, Nemmet P, et al. Atropine cycloplegia: how many instillations does one need?. J Pediatr Ophthalmol Strabismus. 1992;29(3):175-176.

6. Rengstorff RH, Doughty CB. Mydriatic and cycloplegic drugs: a review of ocular and systemic complications. Am J Optom Physiol Opt. 1982;59(2):162-177.

7. Gettes BC, Belmont O. Tropicamide: comparative cycloplegic effects. Arch Ophthalmol. 1961;66(3):336-340.

8. Chua WH, Balakrishnan V, Chan YH, et al. Atropine for the treatment of childhood myopia. Ophthalmology. 2006;113(12):2285-2291.

9. Fan DS, Rao SK, Ng JS, et al. Comparative study on the safety and efficacy of different cycloplegic agents in children with darkly pigmented irides. Clin Exp Ophthalmol. 2004;32(5):462-467.

10. Chua WH, Balakrishnan V, Chan YH, et al. Atropine for the treatment of childhood myopia. Ophthalmology. 2006;113(12):2285-2291. 